\title{
Preparation and evaluation of 2-methoxyestradiol-loaded pH-sensitive liposomes
}

\author{
Suna He ${ }^{*}$, , Bowen Wang ${ }^{1}$, Runfang Zhang ${ }^{1}$, Huanhuan Zhou', Qian Yang1 \\ ${ }^{1}$ Department of Pharmaceutical Sciences, Medical College, Henan University of Science and Technology, Luoyang, People's \\ Republic of China
}

\begin{abstract}
The development and clinical application of 2-methoxyestradiol (2-ME) as a new type of antitumor drug are limited due to its poor solubility, rapid metabolism in vivo, and large oral dosage. 2-ME-loaded $\mathrm{pH}-$ sensitive liposomes (2-ME-PSLs) was prepared containing the lipids, Lipoid E-80 (E-80), cholesteryl hemisuccinate (CHEMS), and cholesterol (CHOL) via thin-film ultrasonic dispersion. First, preparation conditions of 2-ME-PSLs were optimized by orthogonal test. Then 2-ME-PSL was characterized, and the release behavior and stability of 2-ME-PSL in vitro were evaluated. The optimal preparation conditions for 2-ME-PSLs were as follows: 2-ME : E-80+CHEMS 1:15; CHOL: E-80+CHEMS 1:5; ultrasonication time 20 minutes. The mean particle size, PDI, zeta potential, and entrapment efficiency (EE) of 2-MEPSLs were $116 \pm 9 \mathrm{~nm}, 0.161 \pm 0.025,-22.4 \pm 1.7 \mathrm{mV}$, and $98.6 \pm 0.5 \%$, respectively. As viewed under a transmission electron microscope, 2-ME-PSLs were well dispersed and almost spherical. They exhibited significant $\mathrm{pH}$-sensitive properties and were fairly stable when diluted with a physiological solution. In conclusion, 2-ME-PSLs were successfully prepared and possessed a favorable pH sensitivity and good dissolution stability with a normal solution.
\end{abstract}

Keywords: 2-Methoxyestradiol. $\mathrm{pH}$ sensitive liposomes. In vitro release. Stability.

\section{INTRODUCTION}

2-Methoxyestradiol (2-ME) is the physiological metabolite of $17 \beta$-estradiol, catalyzed by catechol- $O$ methyltransferase (COMT) in vivo (Dubey, 2017). It exhibits antitumor effects against a broad spectrum of tumors, especially breast cancer (Salama et al., 2011; Zhao et al., 2017), by anti-angiogenesis, inhibiting proliferation, and/or inducing apoptosis (Massaro et al., 2017; Zou et al., 2018). As a new type of antitumor drug, 2-ME is effective by oral administration. The use of 2 -ME to treat breast cancer is currently in phase II clinical research. However, due to its poor water solubility, rapid metabolism in vivo, and a large oral dosage (Guo et al., 2017; Said et al., 2017), the development and clinical application of its products are limited. Therefore, it is important to focus on the development of new delivery systems for 2-ME.

A liposome, as an artificially prepared spherical vesicle composed of a lamellar phase lipid bilayer, has

\footnotetext{
*Correspondence: S. He. Department of Pharmaceutical Sciences, Medical College, Henan University of Science and Technology, 471003 - 263\# Kaiyuan Road, Luoyang, People's Republic of China. E-mail: hesuna-2008@163.com (ID)
}

great potential application in drug delivery, especially for entrapping hydrophobic drugs to increase drug solubility and targeting (Benson, 2017; Giacometti et al., 2017; Zabielska-Koczywas, Lechowski, 2017). However, liposomes easily leak drugs in physiological environments, which is not a conducive phenomenon because it could not leads to drug aggregation in tumor sites (Wang et al., 2017). In order to improve the liposome therapeutic efficacy, multifunction liposomes have been designed (Dai et al., 2014; Chen et al., 2017; Chi et al., 2017; Dai et al., 2017). Considering that the $\mathrm{pH}$ value of the tumor tissue (about 5.0) is lower than that of the normal tissue, $\mathrm{pH}$-sensitive liposomes have been developed as an efficient system for controlled drug release to improve drug efficacy after intravenous administration (Liang et al., 2017; Lee, Thompson, 2017).

In this study, we prepared 2-ME-loaded $\mathrm{pH}$-sensitive liposomes (2-ME-PSLs), which were stable in the physiological environment, and fused intelligently into the acidic environment of tumor tissues. After optimizing preparation conditions and characterizing the 2-ME-PSLs, we investigated their stability and in vitro release behavior for $\mathrm{pH}$ sensitivity. The study lays down a foundation for 
the future development and application of 2-ME in the treatment of various cancers..

\section{MATERIAL AND METHODS}

\section{Material}

2-methoxyestradiol was purchased from Aikeda Chemical Reagent Co., Ltd. (Sichuan, China), Lipoid E-80 from Lipoid GmbH (Ludwigshafen, Germany), cholesteryl hemisuccinate (CHEMS) and cholesterol (CHOL) from Tokyo Chemical Industry Co., Ltd. (Tokyo, Japan) and high-performance liquid-chromatography (HPLC)-grade methanol from Tianjin Kermel Chemical Reagent Co., Ltd. (Tianjin, China). All other reagents were of analytical grade.

\section{Preparation of 2-ME-PSLs}

The 2-ME-PSLs wasWe prepared via thin-film ultrasonic dispersion method, using the lipids E-80, CHOL and CHEMS in the formulation. Briefly, 2-ME $(10 \mathrm{mg})$ and lipids were dissolved in chloroform and then evaporated at $40^{\circ} \mathrm{C}$ under reduced pressure until the organic solvent was removed to form a thin lipid film. The dried lipid film was hydrated with phosphate-buffered saline (PBS; $10 \mathrm{~mL}$ ) and then sonicated it in a bath-type sonicator at $100 \mathrm{~W}$.

\section{Orthogonal experiment}

In the study, the preparation conditions for 2-MEPSL was optimized PSLs by orthogonal test on the basis of the pre-experiment; the ratios of 2-ME: lipid (A), lipid: cholesterol (B), and ultrasonic time (C) as factors; and particle size as the evaluation index. The lipid was a mixture of E-80 and CHEMS (1:1). The independent factors and the levels were shown in Table I. In order to reduce systematic errors, the experiments were replicated for 3 times and completely randomized. The particle size was expressed as a mean intensity diameter.

\section{Characterization of 2-ME-PSLs}

\section{Particle size and zeta potential}

The particle size, size distribution and zeta potential of 2-ME-PSLs were measured by dynamic light scattering (DLS) analysis using a Malvern Zetasizer Nano ZS90 (Malvern Instruments, Malvern, UK). Each test was performed in triplicate. Results were expressed as mean \pm standard deviation (SD).
TABLE I - Factor and level

\begin{tabular}{lccc}
\hline Level & \multicolumn{3}{c}{ Factor } \\
\cline { 2 - 4 } & $\begin{array}{c}\text { 2-ME: } \\
\text { E80+CHEMS }\end{array}$ & $\begin{array}{c}\text { CHOL: } \\
\text { E80+CHEMS }\end{array}$ & $\begin{array}{c}\text { Sonication time } \\
\text { (min) }\end{array}$ \\
\hline 1 & $1: 10$ & $1: 4$ & 20 \\
2 & $1: 12$ & $1: 5$ & 30 \\
3 & $1: 15$ & $1: 6$ & 40 \\
\hline
\end{tabular}

\section{Morphology investigation}

The morphology of 2-ME-PSLs was observed using a JEM-2010 transmission electron microscope (TEM; JEOL Co., Ltd., Japan) with the negative-staining method. After diluted with an appropriate amount of distilled water, a drop of the sample was deposited on a dedicated copper grid and then stained with $1 \%(\mathrm{w} / \mathrm{v})$ uranyl acetate. The grid was positioned at room temperature and allowed to dry before observation.

\section{Entrapment efficiency}

The encapsulation efficiency (EE) of 2-ME in PSLs was determined according to the literature (Xu et al., 2017) with a little modification. Briefly, 2-ME-PSLs (400 $\mu \mathrm{L})$ was centrifuged in an ultrafiltration tube $(50 \mathrm{kDa}$; Millipore, Massachusetts, US) at $3000 \mathrm{rpm}$ for 30 minutes to separate the water phase. Non-entrapped 2-ME in the water phase was determined directly and total $2-\mathrm{ME}$ in the PSLs was determined after lysing the liposomes with methanol $(1: 5 ; \mathrm{v} / \mathrm{v}) .2-\mathrm{ME}$ in the various samples were detected by HPLC using an LC-20AT pump and SPD-20A UV/VIS detector (Shimadzu Corp., Kyoto, Japan) and a Diamonsil C18 analytical column $(200 \times 4.6 \mathrm{~mm}, 5 \mu \mathrm{m}$; Dikma Technologies, Inc., Foothill Ranch, California, US). The mobile phase was a mixture of acetonitrile and double-distilled water $(50 / 50, \mathrm{v} / \mathrm{v})$ at a flow rate of 1.0 $\mathrm{mL} / \mathrm{min}$ and the detection wavelength was $205 \mathrm{~nm}$. The HPLC method was validated by the studies on precision, accuracy and standard curve (data not shown). The entrapment efficiency (EE) was calculated according to the equation (1):

$$
\mathrm{EE}(\%)=\frac{\mathrm{C}_{\text {total }} \mathrm{V}_{\text {total }}-\mathrm{C}_{\text {water }} \mathrm{V}_{\text {water }}}{\mathrm{C}_{\text {total }} \mathrm{V}_{\text {total }}} \times 100 \%
$$

\section{In vitro release study}

The in vitro release of 2-ME in PSLs was investigated using dialysis in 2 different PBS media ( $0.3 \%$ Tween- 80 , pH $7.4 \pm 0.1$ and $0.3 \%$ Tween-80, pH $5.5 \pm 0.1)$ at $37^{\circ} \mathrm{C}$ (Seong, Yun, Park, 2017). Briefly, $2.0 \mathrm{~mL}$ of 2-ME-PSLs 
was put into a dialysis bag (molecular-weight cutoff $[\mathrm{MWCO}]=8,000-14,000 \mathrm{Da})$, which was soaked in double-distilled water for 24 hours before use. The endsealed dialysis bags were then immersed in $100 \mathrm{~mL}$ release media stirred at $100 \mathrm{rpm}$ at $37^{\circ} \mathrm{C}$. At pre-determined time intervals $(1,2,4,6,8,12,24 \mathrm{~h})$, an aliquot of $0.5 \mathrm{~mL}$ release media was withdrawn and replaced by an equal volume of pre-warmed fresh media. Concentrations of drug in the samples were determined by the HPLC method described above.

\section{Stability study}

Generally, 2-ME-PSLs was given by intravenous injection after dilution with $5 \%$ glucose or $0.9 \%$ sodium chloride injection. Thereforefor, the stability of 2-MEPSLs after diluted with $5 \%$ glucose or $0.9 \%$ sodium chloride injection was evaluated. The physicochemical properties, including appearance, droplet size and drug content, were then investigated.

\section{RESULTS AND DISCUSSION}

\section{Preparation and optimization of 2-ME-PSLs}

The main results of the orthogonal test and range analysis are shown in Table II. The major-minor order that affected particle size was $\mathrm{A}>\mathrm{C}>\mathrm{B}$, that is, the ratio of 2-ME : E-80+CHEMS $>$ sonication time $>$ CHOL: E-80+CHEMS. The optimal levels of 2-ME : E-80+CHEMS and sonication time were 1:15 and 40 minutes, respectively. Particle size decreased as the ratio decreased and as the sonication time increased. The optimal level of CHOL : E-80+CHEMS was 1:5. E-80, CHEMS, and CHOL as the part of the double molecular film affected the formation of liposomes. The optimal conditions for preparing 2-ME-PSLs were as follows: 2-ME, $10 \mathrm{mg}$; E-80+CHEMS (1:1), $150 \mathrm{mg}$; CHOL, 30 $\mathrm{mg}$; distilled water, $10 \mathrm{~mL}$; sonication, 40 minutes.

In this experiment, the liposomes exhibited $\mathrm{pH}$ sensitivity when the acid-responsive CHEMS were anchored to the lipid bilayer. The amount of CHEMS (E$80:$ CHEMS $=1: 1)$ without optimization was according to the literature (Lee, Thompson, 2017). In the orthogonal experimental design, we selected particle size rather than $\mathrm{EE}$ as the evaluation index, mainly because $2-\mathrm{ME}$ is a hydrophobic drug: if the EE was too low, it resulted in drug precipitation, which was visible to the naked eye. Therefore, the particle size was suitable to be as the evaluation index.
TABLE II - The results of orthogonal test

\begin{tabular}{lcccc}
\hline No. & A & B & C & d/nm \\
\hline 1 & 1 & 1 & 1 & 218 \\
2 & 1 & 2 & 2 & 175 \\
3 & 1 & 3 & 3 & 165 \\
4 & 2 & 1 & 2 & 148 \\
5 & 2 & 2 & 3 & 135 \\
6 & 2 & 3 & 1 & 174 \\
7 & 3 & 1 & 3 & 117 \\
8 & 3 & 2 & 1 & 128 \\
9 & 3 & 3 & 2 & 139 \\
\hline $\mathrm{K}_{1}$ & 186 & 161 & 173 & \\
$\mathrm{~K}_{2}$ & 152 & 146 & 154 & \\
$\mathrm{~K}_{3}$ & 128 & 159 & 139 & \\
$\mathrm{R}$ & 58 & 15 & 34 & \\
\hline
\end{tabular}

\section{Characterization of 2-ME-PSLs}

Droplet size distribution and zeta potential are shown in Figure 1. DLS analysis showed the mean particle size, polydispersity index (PDI), and zeta potential of 2-ME-PSLs to be $116 \pm 9 \mathrm{~nm}, 0.161 \pm 0.025$, and $-22.4 \pm 1.7 \mathrm{mV}$, respectively. Figure 2 shows that 2-ME-PSLs were almost spherical in shape and well dispersed without any clustering or aggregation. The particle size was consistent with that determined by DLS analysis, and EE was as high as $98 \%$.

\section{In vitro release study}

In the in vitro release study, $0.3 \%$ Tween- 80 contained in PBS was used to meet the sink condition because of 2-ME's poor solubility. Results are shown in Figure 3. A low level of 2-ME was released from the powder in PBS without Tween- 80 at both $\mathrm{pH} 7.4$ and 5.5 ( $<20 \%$ at 24 hours), further demonstrating the poor solubility of 2-ME. Under the sink condition, the level of 2-ME released from the crude powder increased significantly, but different $\mathrm{pH}$ conditions had no effect on this level. Less than $10 \%$ of $2-\mathrm{ME}$ was released from PSLs in the $\mathrm{pH}$ 7.4 PBS medium within the initial 12 hours, but the level increased markedly after 12 until 24 hours. However, in the pH 5.5 PBS medium, more than $90 \%$ of $2-\mathrm{ME}$ was released from the liposomes during the initial 6 hours, and 2-ME was almost completely released within 8 hours. These release profiles suggested that 2-ME-PSLs had good stability 

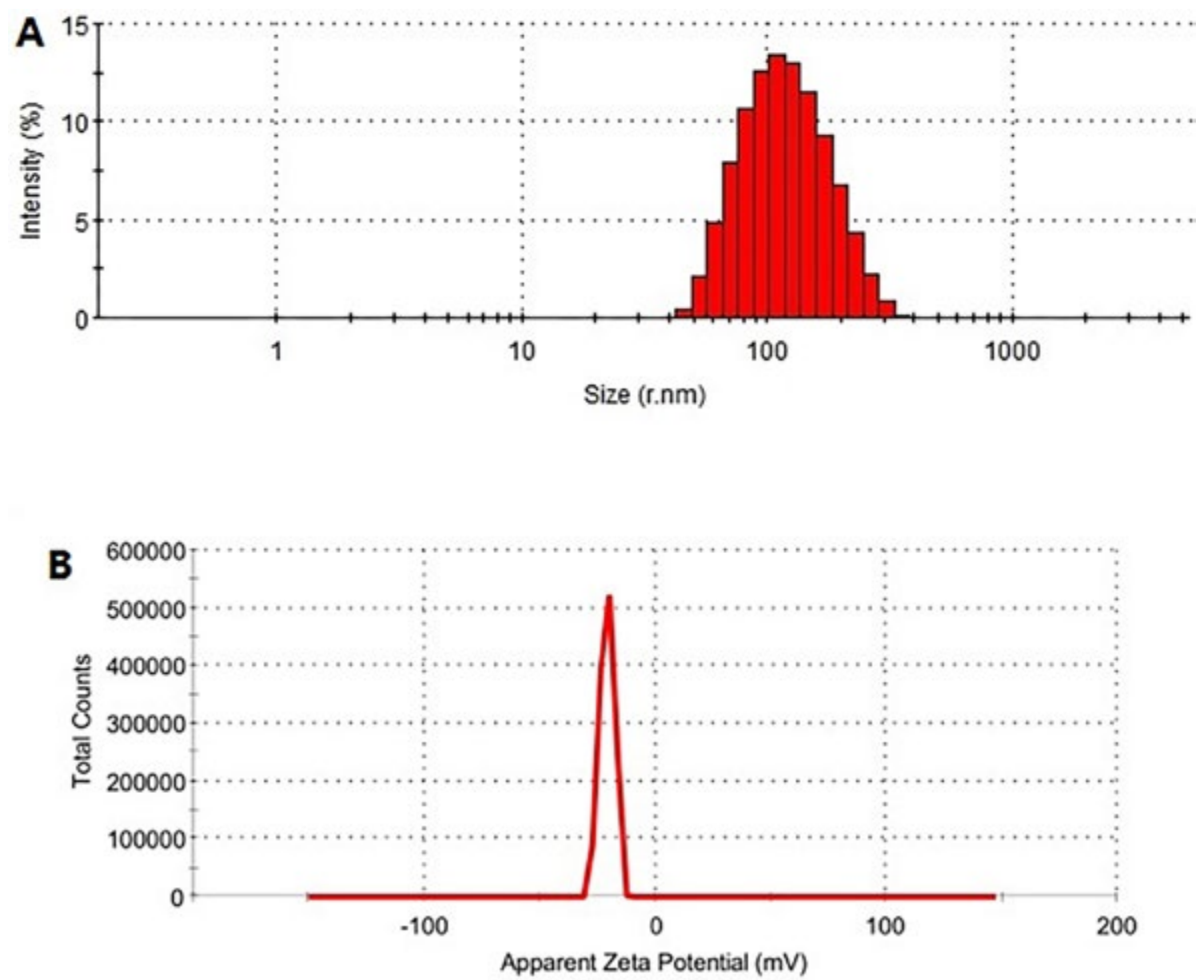

FIGURE 1 - Particle size and zeta potential of 2-ME-PSL.

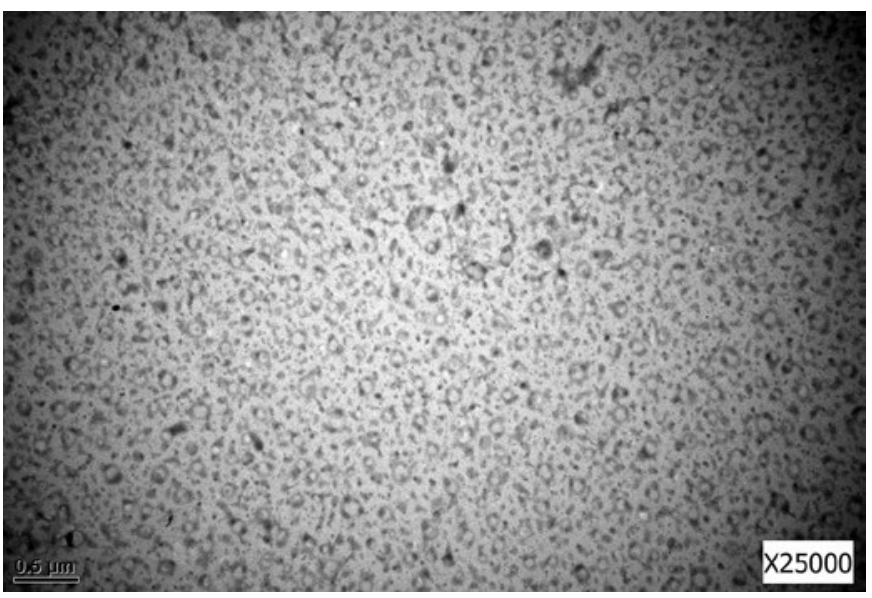

FIGURE 2 - TEM image of 2-ME-PSL.

in a neutral environment ( $\mathrm{pH} 7.4)$ and were released quickly in acidic environments ( $\mathrm{pH}$ 5.5), demonstrating an obvious $\mathrm{pH}$ sensitivity. That is to say, rapid release during the delivery process could be avoided, and the effect of plasma on drug leakage was negligible. The release behavior of 2-ME-PSLs in different media was mainly caused by anchoring acid-responsive CHEMS to the lipid bilayer, which was conducive to 2-ME-PSLs accumulating and exerting their effectiveness in tumor tissues (Monteiro et al., 2017).

\section{Stability study}

As shown in Table III, when the 2-ME-PSLs were diluted with 1-10 times volume of normal saline soluiton, the appearance, particle size, and drug content showed no obvious changes. However, when 2-ME-PSLs were diluted with $5 \%$, the samples were turbid, with subsequent occurrence of precipitation. The results suggested that 2-ME-PSLs were stable when diluted with normal saline solution rather than $5 \%$ glucose. This could be due to the different $\mathrm{pH}$ values of normal saline solution (6.5-7.5) and $5 \%$ glucose (3.5-6.5).

To evaluate the effect of $\mathrm{pH}$ on the stability of 2-ME-PSLs, we confirmed the change in liposome size after incubation with $\mathrm{pH}$ 7.4 PBS and $\mathrm{pH}$ 5.5 PBS. As shown in Figure 4, the size of liposomes gradually increased when incubated with $\mathrm{pH}$ 5.5 PBS, reaching about $1500 \mathrm{~nm}$ at 45 minutes. On the other hand, the 


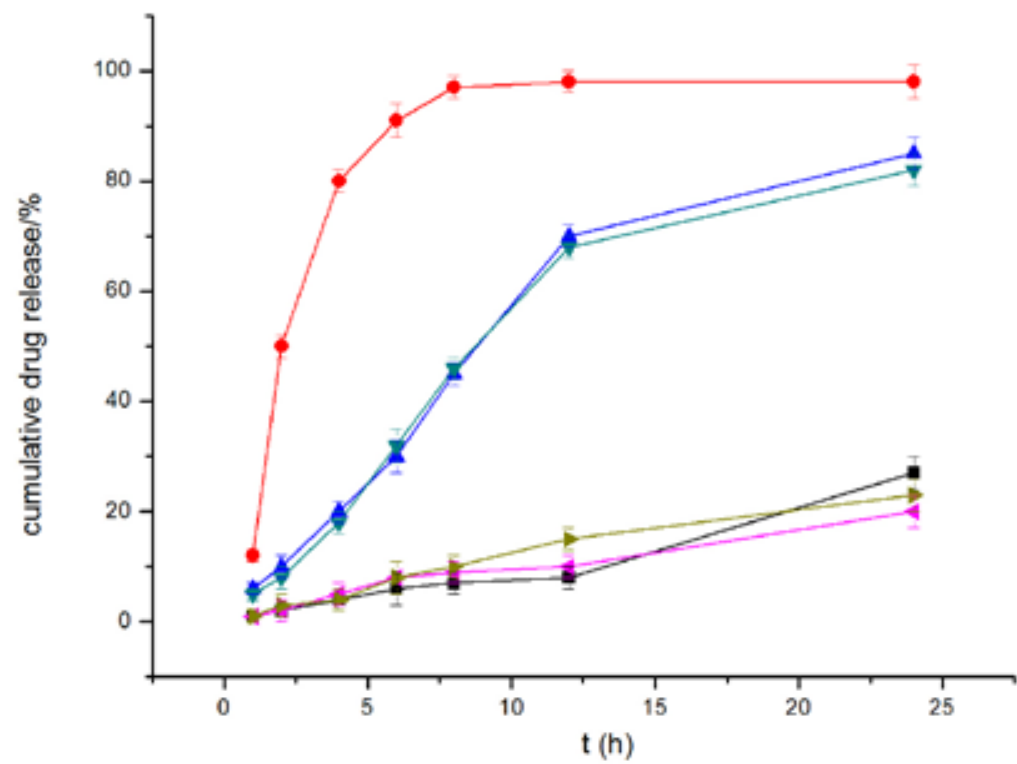

- 2-ME-PSL in pH 7.4 PBS $(0.3 \%$ Tween 80$)-2$-ME-PSL in pH 5.5 PBS $(0.3 \%$ Tween 80$)$
- crude 2-ME powder in pH 7.4 PBS $(0.3 \%$ Tween 80$)-$ crude $2-\mathrm{ME}$ powder in pH $7.4 \mathrm{PBS}$
- crude 2-ME powder in pH 5.5 PBS $(0.3 \%$ Tween 80$) \rightarrow$ crude 2-ME powder in pH 5.5 PBS

FIGURE 3 - In vitro release curves of 2-ME-PSL under different conditions $(\mathrm{n}=3)$.

size of liposomes incubated with pH 7.4 PBS showed no obvious change. Furthermore, we could not observe a normal spherical morphology under TEM for the 2-ME-PSLs incubated with pH 5.5 PBS for 45 minutes. These results suggested that 2-ME-PSLs could lyse at $\mathrm{pH} 5.5$ and then release 2-ME, thus meeting our primary goal that 2-ME-PSLs are stable in blood circulation and quickly release the drug after accumulating in tumor tissues. The structure of CHEMS, which possesses $\mathrm{pH}$ sensitive properties, was the main reason for the $\mathrm{pH}$ sensitivity in 2-ME-PSLs. Because the $\mathrm{p} K_{\mathrm{a}}$ of CHEMS was 5.8, when CHEMS participated in the formation of a liposome membrane, the zeta potential should have been negative in a neutral and/or alkaline environment, consistent with the zeta potential measured above. When CHEMS entered an acidic environment, it was protonated and the carboxyl group was oriented on the surface of the liposomes, resulting in liposome membrane instability and the decomposition of the lipid bilayer (Xiong et al., 2011; Chen et al., 2012). Therefore, 2-ME-PSLs could

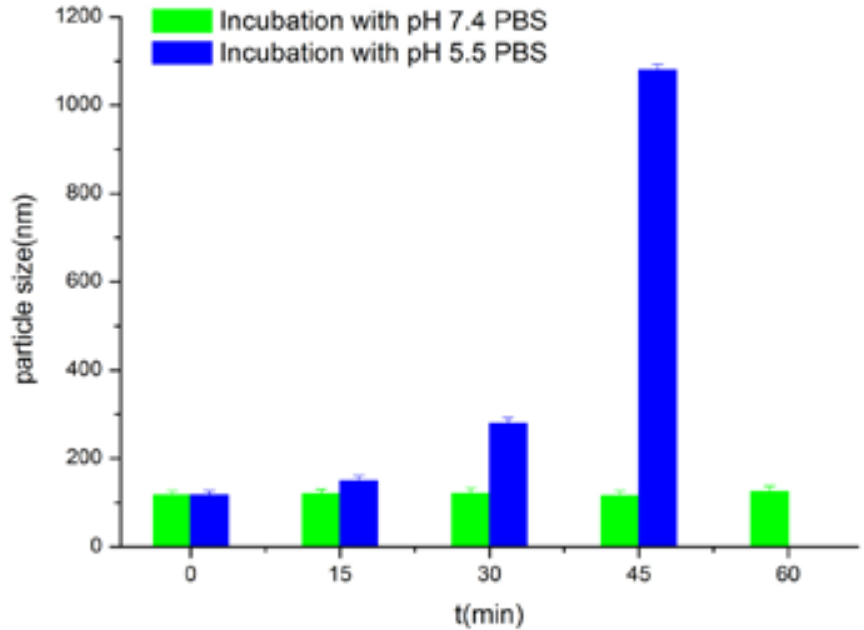

FIGURE 4 - The particle size of 2-ME-PSL incubation with different PBS $(n=3)$.

exist stably in a neutral blood circulation environment. After the liposomes had entered the tumor tissues, their bilayer membrane became unstable and fusogenesis

TABLE III - The results of stability experiments $(n=3)$

\begin{tabular}{ccccc}
\hline Dilution times & Appearance & Particle size/nm & PDI & Drug content (\%) \\
\hline 1 & ivory, opalescence & 129 & 0.21 & 99.1 \\
2 & ivory, opalescence & 119 & 0.17 & 98.2 \\
5 & ivory, opalescence & 117 & 0.19 & 97.1 \\
10 & ivory, opalescence & 122 & 0.19 & 98.4 \\
\hline
\end{tabular}


occurred, thereby promoting drug release and exerting a therapeutic effect. In conclusion, the stability study proved that $\mathrm{pH}$-sensitive 2-ME-PSLs were stable when diluted with normal saline and were therefore suitable for clinical use.

\section{CONCLUSION}

In this study, 2-ME-PSL was successfully prepared with good stability diluted with normal saline and significant $\mathrm{pH}$ sensitivity. From preliminary study, we predicted that 2-ME-PSLs would be stable in blood circulation and would release the drug rapidly after reaching tumor sites, thus achieving the preferable antitumor effect. We intend to further study the pharmacokinetics and pharmacodynamics of 2-ME-PSLs in vivo.

\section{ACKNOWLEDGEMENTS}

This study was funded by the Basic Research Program of Henan Province (No. 18B350002). The authors are grateful for the financial support.

\section{REFERENCES}

Benson HA. Elastic liposomes for topical and transdermal drug delivery. Methods Mol Biol. 2017;1522:107-17.

Chen C, Duan Z, Yuan Y, Li R, Pang L, Liang J, et al. Peptide-22 and cyclic RGD functionalized liposomes for glioma targeting drug delivery overcoming BBB and BBTB. ACS Appl Mater Interfaces. 2017;9(7):5864-73.

Chen D, Sun K, Mu H, Tang M, Liang R, Wang A, et al. pH and temperature dual-sensitive liposome gel based on novel cleavable mPEG-Hz-CHEMS polymeric vaginal delivery system. Int J Nanomedicine. 2012;7:2621-30.

Chi Y, Yin X, Sun K, Feng S, Liu J, Chen D, et al. Redoxsensitive and hyaluronic acid functionalized liposomes for cytoplasmic drug delivery to osteosarcoma in animal models. J Control Release. 2017;261:113-25.

Dai M, Wu C, Fang H, Li L, Yan J, Zeng D, et al. Thermoresponsive magnetic liposomes for hyperthermia-triggered local drug delivery. J Microencapsulation. 2017;34(4):408-15.

Dai W, Yang F, Ma L, Fan Y, He B, He Q, et al. Combined mTOR inhibitor rapamycin and doxorubicin-loaded cyclic octapeptide modified liposomes for targeting integrin alpha 3 in triplenegative breast cancer. Biomaterials. 2014;35(20):5347-58.
Dubey RK. 2-Methoxyestradiol: A 17beta-Estradiol metabolite with gender-independent therapeutic potential. Hypertension. 2017;69(6):1014-6.

Giacometti G, Marini M, Papadopoulos K, Ferreri C, Chatgilialoglu C. trans-Double bond-containing liposomes as potential carriers for drug delivery. Molecules. 2017;22(12):pii:E2082.

Guo X, Chen C, Liu X, Hou P, Guo X, Ding F, et al. High oral bioavailability of 2-methoxyestradiol in PEG-PLGA micellesmicrospheres for cancer therapy. Eur J Pharm Biopharm. 2017;117:116-22.

Liang J, Fang C, Wu W, Yu P, Gao J, Li J, et al. Preparation and properties evaluation of a novel $\mathrm{pH}$-sensitive liposomes based on imidazole-modified cholesterol derivatives. Int J Pharm. 2017;518(1-2):213-19.

Lee Y, Thompson DH. Stimuli-responsive liposomes for drug delivery. Wiley Interdiscip Rev Nanomed Nanobiotechnol. 2017;9(5).

Massaro RR, Faião-Flores F, Rebecca VW, Sandri S, AlvesFernandes DK, Pennacchi PC, et al. Inhibition of proliferation and invasion in 2D and 3D models by 2-methoxyestradiol in human melanoma cells. Pharmacol Res. 2017;119:242-50.

Monteiro LOF, Fernandes RS, Oda CMR, Lopes SC, Townsend DM, Cardoso VN, et al. Paclitaxel-loaded folate-coated long circulating and $\mathrm{pH}$-sensitive liposomes as a potential drug delivery system: A biodistribution study. Biomed Pharmacother. 2017;97: 489-95.

Said A, Zaki R, Eldin RA, Nasr M, Azab S, Elzankalony Y. Efficacy of Intravitreal injection of 2-Methoxyestradiol in regression of neovascularization of a retinopathy of prematurity rat model. BMC Ophthalmol. 2017;17(1):38.

Salama S, Diaz-Arrastia C, Patel D, Botting S. 2-Methoxyestradiol, an endogenous estrogen metabolite, sensitizes radioresistant MCF-7/FIR breast cancer cells through multiple mechanisms. Int J Radiat Oncol Biol Phys. 2011;80(1):231-9.

Seong JS, Yun ME, Park SN. Surfactant-stable and pHsensitive liposomes coated with N-succinyl-chitosan and chitooligosaccharide for delivery of quercetin. Carbohydr Polym. 2017;181:659-667. 
Wang M, Zhao T, Liu Y, Wang Q, Xing S, Li L, et al. Ursolic acid liposomes with chitosan modification: Promising antitumor drug delivery and efficacy. Mater Sci Eng C Mater Biol Appl. 2017;71:1231-40.

Xiong S, Yu B, Wu J, Li H, Lee RJ. Preparation, therapeutic efficacy and intratumoral localization of targeted daunorubicin liposomes conjugating folate-PEG-CHEMS. Biomed Pharmacother. 2011;65(1):2-8.

Xu H, Zhang L, Li L, Liu Y, Chao Y, Liu X, et al. Membraneloaded doxorubicin liposomes based on ion-pairing technology with high drug loading and $\mathrm{pH}$-Responsive property. AAPS PharmSciTech. 2017;18(6):2120-30.
Zabielska-Koczywas K, Lechowski R. The use of liposomes and nanoparticles as drug delivery systems to improve cancer treatment in dogs and cats. Molecules. 2017;22(12):pii:2167.

Zhao H, Jiang H, Li Z, Zhuang Y, Liu Y, Zhou S, et al. 2-Methoxyestradiol enhances radiosensitivity in radioresistant melanoma MDA-MB-435R cells by regulating glycolysis via HIF-1alpha/PDK1 axis. Int J Oncol. 2017;50(5):1531-40.

Zou X, Zhang L, Yuan J, Yang C, Wu Z, Song J, et al. Endogenous hormone 2-methoxyestradiol suppresses venous hypertension-induced angiogenesis through up- and downregulating p53 and id-1. J Cell Mol Med. 2018;22(2):957-67.

Received for publication on $19^{\text {th }}$ March 2018 Accepted for publication on $12^{\text {th }}$ June 2018 